\title{
Microfluidic-aided fabrication of nanoparticles blend based on chitosan for a transdermal multidrug delivery application
}

\author{
Mohammad Shamsi ${ }^{\mathrm{a}}$, Payam Zahedi $^{\mathrm{a}}{ }$, Hedayatollah Ghourchian $^{\mathrm{b}}$, Sara Minaeian ${ }^{\mathrm{c}}$ \\ a Nano-Biopolymers Research Laboratory, School of Chemical Engineering, College of \\ Engineering, University of Tehran, P. O. Box: 11155-4563, Tehran, Iran \\ ${ }^{b}$ Institute of Biochemistry and Biophysics (IBB), University of Tehran, Tehran, Iran \\ ${ }^{c}$ Antimicrobial Resistance Research Centre, Rasoul-e-Akram Hospital, Iran University of Medical \\ Sciences, Tehran, Iran
}

To whom correspondence should be addressed:

Email: phdzahedi@ut.ac.ir (P. Zahedi)

Tel: +98 (21) 61113062

Fax: $+98(21) 66957784$

ORCID: http://orcid.org/0000-0001-6636-4534 


\begin{abstract}
The aim of this work was to provide a microfluidic-aided fabrication of nanoparticles based on chitosan/poly (N-isopropylacrylamide-co-acrylic acid)/cellulose laurate [CS/P(NIPAAm-co$\mathrm{AAC} / \mathrm{CL}$ ] blend for transdermal multidrug delivery applications. The scanning electron microscopy (SEM) and dynamic light scattering (DLS) results showed that the diameter sizes of samples were in the range from 200 to $300 \mathrm{~nm}$ along with a narrow size distribution. Also, the CS-based nanoparticles containing tretinoin and clindamycin phosphate prepared using microfluidic technique exhibited a sustained control release of the drugs as well as minimum inhibitory and bactericidal concentrations compared to the samples fabricated via bulk mixing method. The thermal stability of the drugs loaded nanoparticles revealed a reduction in degradation for those fabricated by using microfluidic technique at $45^{\circ} \mathrm{C}$ for one month. Afterward, the in vivo assessments confirmed that by applying the microfluidically generated nanoparticles containing two drugs, a declined superficial reddening (erythema) and suitable transdermal permeation as well as residency were happened with respect to the those samples prepared via bulk mixing method and also the drugs solution alone. Finally, these CS-based nanoparticles showed sufficient potential used for transdermal multidrug delivery applications.
\end{abstract}

Keywords: nanoparticle; microfluidic method; chitosan; transdermal drug delivery 


\section{Introduction}

In the past decade, a wide range of natural and synthetic biopolymers such as chitosan (CS), poly (lactic acid) (PLA), poly (lactic-co-glycolic) acid (PLGA), etc., have been extensively considered to produce the nanoparticles with tunable properties as drug delivery carriers by the use of highly efficient microfluidic (MF) technique [1-4]. CS is an N-deacetylated derivatives of chitin that can be obtained from shrimp shell or arthropods and it has abundantly considered in a wide variety of researches. This is due to unique properties of CS in terms of inherently antimicrobial, low toxicity, high efficiency entrapment of either hydrophilic or hydrophobic drugs because of the tunable functional groups e.g., amines $\left(-\mathrm{NH}_{2}\right)$ and/or hydroxyl $(-\mathrm{OH})$ onto its surface [2, 5-7]. Furthermore, by controlling the shape and size of CS-based nanoparticles, their adhesion to jelly-like mucus in gastrointestinal tract and also skin appendices can be enhanced $[8,9]$. Besides biopolymers applications especially CS, thermo-sensitive polymers are recently considered a great deal of attention by researchers $[10,11]$. Poly $(N-$ isopropylacrylamide) [P(NIPAAm)] is a well-known polymer with a lower critical solution temperature (LCST) of $32^{\circ} \mathrm{C}$ which extensively uses in biological applications. By copolymerizing NIPAAm with acrylic acid (AAC) monomer, P(NIPAAm-co-AAC), the LCST of P(NIPAAm) can be increased to body's temperature for getting a higher performance as a drug delivery vehicle through in vitro applications [12].

Blending CS with P(NIPAAm-Co-AAC) can provide an interesting synthesized nanoparticles for topical drug delivery purposes. Because of hydrophilic nature of both P(NIPAAm-Co-AAc) and CS, the addition of poorly water-soluble drugs to them does not allow. To overcome this drawback, cellulose laurate $(\mathrm{CL})$ owing to its hydrophobic property can be added to this blend, as a result, the nanoparticles blend based on CS/P(NIPAAm-Co-AAC)/CL has much more capability for the entrapment of both hydrophilic and hydrophobic drugs molecules.

Despite chemical crosslinking is the standard and efficient procedure for stabilizing CSbased nanoparticles against dissolving in aqueous solutions [13], herein; we have produced them by the use of MF device as the improved bottom-up precipitation technique. The major factor can influence on this system is $\mathrm{pH}$ variations by adding basic solutions [2]. On the other 
hand, one of the main differences between the bulk mixing (BM) and MF methods is the polydispersity index values of the produced nanoparticles in each system. The formation of selfassembled nanoparticles in BM technique leading a broad polydispersity in their diameters size which results in an extent of inappropriate physical and chemical properties with poor performance. In contrast, on-chip fabrication by means of MF method provides the selfassembly of nanoparticles with a minimum size in their diameter and as a result, a narrow polydispersity index is attained [14]. These microfluidically generated nanoparticles with their unique properties are influenced by the formation of a stabilized narrow regime via hydrodynamic flow focusing phenomenon. This controlled mixing regime can be adjusted by manipulating the solvent and anti-solvent flow rates ratios and their solutions concentrations. The optimization of these variables in MF devices enables us to fabricate versatile nanoparticles in which the encapsulation efficiency of drugs molecules as well as their release profile can increase and control, respectively [1].

The application of CS-based nanoparticles generated by MF technique as drugs carriers can be consider to use them in the treatment of skin disorders such as acne vulgaris that can be rationality enumerated for the development of transdermal drug delivery systems. Although acne vulgaris or in other words, simply acne, provides no severe conditions for human worldwide in which their life be susceptible to danger, its adverse side effects for a long time enable to have a series of disorders such as mental illness, decreased self-confidence, emotional depression, etc. [15-17]. Acne is a dermatologic disorder which belongs to pilosebaceous units, as its etiology has not exactly been discovered so far. However, acne pathogenesis is a multifactorial process and includes sebum production and inflammation, blockage of pilosebaceous canal by hyperkeratosis cells as well as Propionibacterium acnes (P.acnes) proliferation and the presence of chemotactic lesions followed by hormone variations $[18,19]$. To overcome acne vulgaris, a number of topical and systemic drug therapies or pharmacotherapies are available $[20,21]$. Among them, multidrug methods for treating acne have proved much more efficiency and safety solutions compared to the other techniques. These new methods have shown a great promise to accelerate the treatment process and reduce the therapeutic time of acne owing to using drugs with complementary mechanisms of 
action [22, 23]. The most common drugs used for this trend are topical retinoids and antibiotics. The retinoids are comedolytic and anti-inflammatory drugs, whereas the antibiotics are antimicrobial drugs along with gentle comedolytic properties [24, 25]. The simultaneous use of these two categories of drugs enables to provide a synergistic effect on the acne treatment. Also, the multidrug formulation including tretinoin (Tre) and clindamycin phosphate (Clin) is more easily sustained via patients compared to Tre lonely, which probably refers to the skin sensitivity decrease owing to using Clin as an antibiotic [26].

Over the past half-decade, the development of various drug delivery strategies has been potentially studied to overcome the shortcomings of the topical anti-acne drugs administrations [27-30]. Despite using the novel multidrug delivery as an efficient therapeutic method for acne vulgaris, many restrictions have been yet remained in this filed. However, the selective recognition of acne sites by means of drugs to release themselves are the fundamental problems. Moreover, the penetration of drugs molecules through skin layers is the main obstacle interfering their bioactive efficiencies as a function of time. Although, the drugs molecules diffusion in the skin layers leads to treat acne vulgaris, their absorption via blood circulation in the body may occur remarkable adverse side effects. Therefore, fabricating a drug delivery system to have penetration selectively through the skin layers and also to transfer effectively the drugs to sites infected with P.acnes bacteria as far as causing a minimum skin irritation is essential.

The objective of this work is to provide a multidrug-loaded nanoparticles based on a special CS blend containing Tre and Clin by using MF technique as a continuous and repeatable process for reduction of adverse side effects such as erythema in transdermal drug delivery applications. Accordingly, a series of in vitro and in vivo experiments have been carried out in order to evaluate the performance of microfluidically fabricated CS/P(NIPAAm-Co-AAc)/CL blend nanoparticles containing Tre and Clin drugs and compared their efficiencies with those samples prepared by traditional BM method and drugs solution alone. Moreover, the obtained results by using SEM, DLS and ultraviolet (UV-vis) spectrophotometry as well as in vivo animal models are investigated and discussed in detail. 


\section{Experimental}

\subsection{Materials}

Poly (N-isopropylacrylamide-co-acrylic acid) [P(NIPAAm-co-AAc)] and also cellulose laurate (CL) were synthesized according to our previous work [12]. Chitosan (CS) (a biological macromolecule and random copolymer of D-glucosamine and $\mathrm{N}$-acetyl-D-glucosamine, average molecular weight of $37 \mathrm{kDa}$ and deacetylation degree of $85 \%$ ) was purchased from SigmaAldrich Co., Pillsburg, The Netherlands. Clindamycin phosphate (Clin) (a white powder and water-soluble drug) and tretinoin (Tre) (a poorly water-soluble drug with 99\% purity extracted from fruits and vegetables) were obtained from Hunan Arshine Biotechnology Co., Ltd., and Xi'an Huilin Bio-tech Co., Ltd., China, respectively. The polymer gel (grade Carbopol ${ }^{\circledR} 934 \mathrm{P}$ NF) was supplied from Lubrizol Advanced Materials Inc., United States. All the other chemicals were analytical reagent grades and used without further purification.

\subsection{Microfluidic device fabrication}

The MF device used in this work was made from poly (methyl methacrylate) (PMMA) and its details as well as assembled chip are represented in Figure 1(a, b). As it is seen, the MF chip has several micro-channels (200 $\mu \mathrm{m}$ in width and $50 \mu \mathrm{m}$ in depth) for the solutions streams [Fig. 1(a)]. In this instrument, one inlet flow (inlet 1) in central channel and the other inlets flows (inlets 2 and 3 ) in lateral channels were embedded. Eventually, after reaching two streams each other, a hydrodynamic flow focusing through the mixing region was formed followed by the nanoparticles on the basis of precipitation technique are produced [Fig. 1(b, c)]. For manufacturing the MF chip with channels in required dimensions, two PMMA sheets with $4 \mathrm{~mm}$ in thickness were utilized. On one hand, the one sheet was considered for etching via $\mathrm{CO}_{2}$ laser apparatus (Guangdong Han's Yueming Laser Tech. Co., Ltd., Taiwan) to provide the microchannels with T-junction pattern that predesigned by using an AutoCAD software. On the other 
hand, the another sheet was applied as the bottom layer to adhere the etched layer [31]. The inlets and outlet places for injecting the dispersed and continuous phases were connected by common syringe needles with gauge of $1 \mathrm{~mm}$ and fixed with cyanoacrylate liquid adhesive. Owing to prevention from liquids leakage, two upper and lower PMMA sheets were fasten tightly by means of fine screws [32]. Finally, the connections between the channels inlets, outlet and syringe needle tips were set on the programmable syringe pumps (New Era Pumps Systems Inc., United States) using silicone rubber tubes.

2.3. Preparation of the dual drug-loaded nanoparticles based on CS/P(NIPAAm-Co-AAc)/CL blend

First, weighed CS powder was added to acetic acid solution ( $\mathrm{pH} \mathrm{4.5)} \mathrm{and} \mathrm{stirred} \mathrm{overnight} \mathrm{to}$ provide the CS solution with 0.1 (\% w/v) concentration. Subsequently, 0.1 (\% w/v) of $\mathrm{P}$ (NIPAAm-co-AAc) containing Clin (1\%) dissolved in distilled water and also $0.1(\% \mathrm{w} / \mathrm{v})$ of $\mathrm{CL}$ including Tre (0.025\%) dissolved in methanol were prepared, filtered and then blended together (1:1). Subsequently, the nanoparticles samples consisted of CS/P(NIPAAm-Co-AA)/CL blend (6:1:1 ratio) containing two drugs were prepared using BM and MF methods, separately.

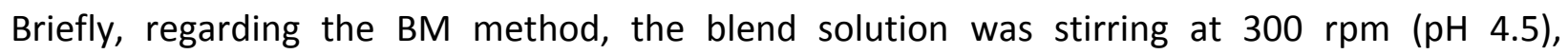
simultaneously, sodium hydroxide $(\mathrm{NaOH})$ solution $(1 \mathrm{M})$ was added drop wise to the solution. The procedure was continued until pH 7.4 was attained for the nanoparticles formation. On the other hand, in regard to the MF technique, by the use of micro-syringe pumps, the blend solution ( $\mathrm{pH} 4.5)$ was injected to the middle channel (inlet 1) and $\mathrm{NaOH}$ solution (pH 9) was pumped to the adjacent channels (inlets 2 and 3 ) with different flow rate ratios $\left(Q_{\text {polymer }} / \mathrm{Q}_{\mathrm{NaOH}}\right)$ of $0.05,0.1$ and 0.2 [Fig. 1(b,c)]. In this way, the polymer blend and $\mathrm{NaOH}$ solutions were reached each other through cross-sectional location on the MF chip and led to the formation of drug-loaded nanoparticles in the diffusion region (the end of middle channel) at $\mathrm{pH} 7.4$ according to the hydrodynamic flow focusing phenomenon [2]. The obtained samples were denoted as follows: BMCSNP, MFCSNP0.05, MFCSNPO.1 and MFCSNP0.2.

\subsection{Preparation of the gel samples}


The gels containing BMCSNP and MFCSNP0.05 as well as drugs solution alone with and without fluorescence dye marker were prepared as the following recipe: weighed Carbopol ${ }^{\circledR} 934 \mathrm{P}$ NF $(1 \% \mathrm{w} / \mathrm{v})$ was dispersed into $100 \mathrm{~mL}$ of deionized water under a constant stirring [33]. Simultaneously, the predetermined amounts of multidrug-loaded nanoparticles and also the drugs solution containing $1 \%$ of Clin and $0.025 \%$ of Tre were inserted to the gel mixture. After the gels formation, they were completely dried at room temperature which were denoted by BMCSNP-gel, MFCSNP0.05-gel, DYMFCSNP0.05-gel, DYDS-gel and DS-gel.

\subsection{Polydispersity, zeta potential, morphology and drug loading efficiency}

The nanoparticles size distribution and their zeta potential results were measured by using dynamic light scattering (DLS) and zeta potential analyzer respectively by means of Zeta plus, Brookhaven, United States. After freeze-drying of the nanoparticles solutions and gold sputtering process, their morphology was observed using a SEM (Philips XL30, Eindhoven, The Netherlands). Due to investigating the drugs loading efficiency of the nanoparticles, they were immersed into phosphate buffer solution (PBS, pH 7.4) containing 0.1 (\% w/v) Tween ${ }^{\circledR} 80$. Thereafter, the solutions containing the nanoparticles were centrifuged at $18000 \mathrm{rpm}$ for 10 min and the concentrations of Clin and Tre in the supernatant were measured by a UV-vis spectrophotometry (Termo 2000, Nanodrop software, United States) at maximum wavelengths of $210 \mathrm{~nm}$ and $344 \mathrm{~nm}$, respectively. The drugs loading efficiency of BMCSNPs and MFCSNPs was calculated based on the Eq. (1) as follows:

Drug loading efficiency $=\frac{W_{0}-W_{s}}{W_{0}}$,

where, ' $W_{0}$ ' and ' $W_{s}$ ' are the amounts of drugs before the nanoparticles formation and those amounts in the supernatant, respectively.

\subsection{Antibacterial assay and in vitro drug release}

To investigate the antibacterial evaluation of the samples against P.acnes (a Gram-positive bacterium) and calculate the minimum inhibitory concentration (MIC) as well as the minimum bactericidal concentration $(\mathrm{MBC})$, the disc diffusion method and turbidimetry were used, 
respectively [34]. Regarding the disc diffusion method, five samples including BMCSNP and MFCSNP0.05 (with and without drugs) and the drugs solution alone were prepared $(0.7 \mathrm{~cm}$ in diameter) and located at a Petri-dish containing Luria-Bertani medium solid agar. Then, they were incubated for $24 \mathrm{~h}$ at $37^{\circ} \mathrm{C}$ and the inhibitory zones were photographed and reported. On the other hand, to calculate MIC and MBC values, a series of tubes containing $5 \mathrm{~mL}$ of Muller Hinton broth (MHB, Difco, United Kingdom) were autoclaved at $121^{\circ} \mathrm{C}$ for $15 \mathrm{~min}$. The dried BMCSNPs and MFCSNPs with and without the drugs were dispersed into distilled water and then acetic acid (0.25\%) was added drop wise to the dispersed aqueous until pH 5 was attained. Then, $5 \mathrm{~mL}$ of each solution containing the nanoparticles $\left(1 \mathrm{mg} \mathrm{mL}^{-1}\right)$ was inserted in $1^{\text {st }}$ tube. In continuous, $2.5 \mathrm{~mL}$ of the solution in $1^{\text {st }}$ tube was transferred to $2^{\text {nd }}$ tube and the solution volume was increased to $5 \mathrm{~mL}$ by adding distilled water to make the solution concentration of $0.5 \mathrm{mg} \mathrm{mL}^{-1}$. This procedure was done two another times for making third and fourth tubes that the solution concentrations were adjusted in the next tube in which a half concentration was obtained with respect to the previous tube. Thereafter, under sterile condition, $50 \mu \mathrm{L}$ of asprepared and fresh suspension containing P.acne bacterium was added to the each tube. In general, the observations based on the bacterium growth or in other words, the turbidity content of those solutions was carried out to calculate MIC values [35-37]. Furthermore, MBC values as the solution concentration of CS by which $99.9 \%$ of the bacteria are killed, were measured. These tests were done in the presence of agar medium containing eosin-methylene blue (EMB).

In vitro drug release of the BMCSNPs and MFCSNPs containing Clin and Tre was investigated using a dialysis bag method. After calculating the calibration curves of each drug on the basis of Beer-Lambert law, $100 \mathrm{mg}$ of the dried nanoparticles were inserted in cellulose-based dialysis bags (Sigma-Aldrich Co., The Netherlands) with a molecular mass cut off of $12 \mathrm{kDa}$ and then they immersed separately into $50 \mathrm{~mL}$ of acetate buffer solution (AcBS, pH 5.5) and PBS (pH 7.4) containing $0.1(\% \mathrm{w} / \mathrm{v})$ Tween ${ }^{\circledR} 80$. The release environments were stirred at $100 \mathrm{rpm}$ at temperature of $37 \pm 0.1^{\circ} \mathrm{C}$. The cumulative release of drugs was done by sampling from their supernatants at predetermined time intervals and subsequently the fresh AcBS and PBS were 
replaced to keep constant the total solutions volumes $(50 \mathrm{~mL})$. The drugs concentrations at each time were calculated based on the calibration curve equations as follows:

For Clin: $A=33.2 \times c$,

For Tre: $A=0.66 \times c$,

where ' $A$ ' is the absorbance of Clin and Tre, and also ' $C$ ' is the drug concentration in the release environment.

\subsection{Thermal stability study}

A long-term thermal stability of the nanoparticles samples containing Tre and Clin drugs was studied at two different temperatures of $25 \pm 1^{\circ} \mathrm{C}$ and $45 \pm 1^{\circ} \mathrm{C}$ during 30 days. Four samples including Clin-MFCSNP0.05, Tre-MFCSNP0.05, Clin-BMCSNP and Tre-BMCSNP were placed in the bottles covered completely with an aluminum foil to protect them against light. After each ten days interval, the weighed samples were suspended in PBS (pH 7.4) for $72 \mathrm{~h}$, subsequently the residual drugs contents in the supernatants were measured by using a the UV-vis spectrophotometry at the related absorbed wavelengths of the drugs. All the experiments were done at least three times and the average with the standard deviation of less than $5 \%$ was recorded.

\subsection{In vivo skin sensitivity, permeation and residency studies}

Regarding the skin sensitivity while using the gel samples, three different formulations in terms of BMCSNP-gel, MFCSNP-gel and DS-gel containing Clin and Tre drugs were selected to study their behaviors onto the skin of rabbit according to the Draize test [38]. This experiment was carried out and followed the ethical permission of Iran University of Medical Sciences (IUMS). Accordingly, some male rabbits $(1.9-2 \mathrm{~kg}, \mathrm{n}=3)$ were selected and sectioned into four groups. The hairs on the back of each rabbit with area of $2 \times 2 \mathrm{~cm}^{2}$ were removed by using a depilatory cream. As mentioned before, the gels have $1 \%$ of Clin and $0.025 \%$ of Tre; therefore, $0.5 \mathrm{~g}$ of each gel was weighed to rub them onto the surfaces of skin for irritation studies. Four groups of the rabbits' skin treatments were considered as follows: the skins have received no 
drugs (control) (group 1), the skins were treated with DS-gel (group 2), BMCSNP-gel (group 3) and MFCSNP0.05-gel (group 4). Also, the following scoring system according to the sensitivity level of treated skin was applied to report: skins without inflammation (score 0), skins with low inflammation (light pink-color, score 1), skins with medium inflammation (dark pink-color, score 2) and skins with severe inflammation (red-color, score 3)[38].

The skin permeation level of the MFCSNP0.05-gel, BMCSNP-gel and DS-gel samples containing the drugs were evaluated by abdomen skin of wistar rats (200-250 g, n=3). The Franz' diffusion cell from glass material manufactured in house, with the effective permeation area of $3.14 \mathrm{~cm}^{2}$ and a receptor chamber with $15 \mathrm{~mL}$ in volume was used in this test. The skins were placed between the receptor chamber and donor section at which the stratum corneum (horny layer) of the skins was in the exposure of the donor section. In other words, the gels should be directly contacted hairs follicles of the skins. The receptor chamber was containing 15 $\mathrm{mL}$ of PBS $(\mathrm{pH} 7.4)$ and Tween ${ }^{\circledR} 80(0.1 \% \mathrm{w} / \mathrm{v})$ with continuously stirring at $32 \pm 1^{\circ} \mathrm{C}$. This experiment was done during $24 \mathrm{~h}$ while putting $0.5 \mathrm{~g}$ of each gel onto the skins in the donor section. At predetermined time intervals, the sampling with amount of $400 \mu \mathrm{L}$ from the receptor chamber was taken and the absorbance was measured using the UV-vis spectrophotometry. After each sampling, $400 \mu \mathrm{L}$ of fresh PBS was immediately added to the chamber for keeping constant the total volume.

Due to determination of residency amount of the drugs loaded into nanoparticles through the skins follicles, the stratum corneum regions onto the surfaces of skins used in the previous section, were completely taken via striping tapes. Briefly, the skins surfaces were first washed with PBS three times and drip-dried using filter papers. Then, to remove the stratum corneum with amount of $100 \mu \mathrm{g} \mathrm{cm}^{-2}$, ten striping tapes were used for ten times. Finally, the tapes were immersed into PBS (pH 7.4) containing 0.1 (\% w/v) Tween ${ }^{\circledR} 80$ in order to measure the amount of the drugs residency through the skins layers by using the spectrophotometry.

For visual confirmation, a fluorescence microscopy (Axioskop 2 plus, Ziess, Germany) was utilized to study the presence of drugs at the middle regions of skins layers. As aforementioned, the gels containing the nanoparticles prepared using MF method and also the drugs solution 
alone colored via a fluorescence dye were topically administered on the skins surfaces of rats. Subsequently, the cross-sectional areas of the skins layers treated with the gels were cut by using a microtomy process until a thin layer was obtained. The cutaneous layers were studied under the fluorescence microscopy and their bulk areas were photographed in the presence and absence of common light and reported.

\section{Results and Discussion}

\subsection{Characterization of CS/P(NIPAAm-Co-AAC)/CL blend nanoparticles}

In general, the nanoparticles produced using BM technique are under the heterogenic reaction conditions, consequently it is difficult to control their sizes and polydispersity. In contrast to $B M$ technique, in the MF method, $Q_{\text {polymer }} / Q_{N a O H}$ is a key factor for controlling the average diameter size and zeta potential of nanoparticles. This can be related to the presence of laminar flow predominantly formed owing to very low Reynold's number $(R e<100)$ through the micro-channels. The interfacial forces between the solutions in the diffusion region of MF chip have the effective parameter in controlling the final physical properties of nanoparticles. In other words, these interfacial forces can rise while increasing the solutions flow rates leading a reduction of nanoparticles size and their size distribution $[1,2,14]$.

Fig. 1(c) illustrates the optical microscopy photographs of hydrodynamic flow focusing phenomenon with different flow ratios $\left(Q_{\text {polymer }} / Q_{\mathrm{NaOH}}\right)$ including $0.05,0.1$ and 0.2 . As it is evident, by increasing the $\mathrm{NaOH}$ flow rate, the precipitation region of two phase solutions was concentrated and their distance become smaller remarkably through the mixing length in the microfluidic chip. According to this event, a series of CS-based nanoparticles were formed with different sized and polydispersity. Figure 2(a-d) depicts the DLS results and as it is seen from Fig. 2(b-d), the samples including MFCSNP0.05, MFCSNP0.1 and MFCSNP0.2 have shown average diameters of $208 \pm 35,220 \pm 82$ and $255 \pm 78 \mathrm{~nm}$, respectively. Also, the polydispersity curves of these samples were approximately narrow. Moreover, the BMCSNPs have a broad size distribution in diameter with an average of $480 \pm 298 \mathrm{~nm}$ [Fig. 2(a)]. By comparing these results, it could be concluded that the MF method has higher performance with respect to BM technique for the production of CS-based nanoparticles with a fine size and narrow 
polydispersity. Therefore, the DLS results showed that the best solutions flow rate ratio of CS/P(NIPAAm-CO-AAC)/CL to $\mathrm{NaOH}$ was 0.05 which led to the most narrow polydispersity for the prepared nanoparticles [Fig. 2(b)].

For further investigation, Figs. 2(e) and 2(f) show the morphology of the BMCSNP and MFCSNP0.05 samples, respectively. The SEM micrograph images were also confirmed those results obtained from DLS test. According to Fig. 2(e), the nanoparticles prepared using BM method (BMCSNPs) have undergone the aggregated structures and collapsed particles with sizing 182 to $778 \mathrm{~nm}$. In contrast, the nanoparticles denoted by MFCSNP0.05 have shown a uniform morphology with spherical shapes that their overall diameters sizes were less than 300 $\mathrm{nm}$ [Fig. 2(f)]. These observations resulted in the MF technique as compared to BM method was a more precise process in controlling the nanoparticles size and their polydispersity. In other words, the MF method is an efficient tool in order to fabricate nanoparticles based on CS containing the drugs along with small sizes in diameter resulting an appropriate morphology.

As it is illustrated in Figure 3(a-b), because of the lower surface charges of the nanoparticles produced using MF technique compared to those samples fabricated via BM method, the loading efficiency of Clin was very high. This trend was also seen for Tre drug with smaller variations [Fig. 3(a)]. Although, increasing the flow rate ratios from 0.05 to 0.2 was leveled up the loading efficiency of drugs and decreased surface charges of the nanoparticles, the low flow rate ratio of 0.05 was selected for further investigations because of the negative charge of hairs onto the skin surfaces in which a suitable interaction can be provide between the nanoparticles and hairs follicles.

\subsection{Antibacterial assay and in vitro drug release of the samples}

Figure 4(a) depicts the antibiogram model of the samples including the drugs solution (DS), BMCSNP and MFCSNP0.05 with and without Tre and Clin drugs at concentrations of $2 \mu \mathrm{gL}^{-1}$ against P.acnes bacteria. As it can be observed, all the samples containing CS-based nanoparticles (No. 2, 3, 4 and 5) showed suitable bacterial inhibitory zones with approximately the same areas that this reason was related to the inherently antimicrobial property of CS. The sample with No. 1 referred to the DS exhibited no antibacterial property which confirmed that 
the drugs could not inhibit the bacteria growth. Another important information was found based on MIC and MBC values of the samples. According to Table 1(a), these contents are shown for the samples in terms of DS and BMCSNP as well as MFCSNP0.05 with and without Tre and Clin drugs. The MIC values was minimal for the MFCSNP0.05 containing the drugs that was due to the small sizes and uniformity of the nanoparticles produced using MF technique $[36,37]$. The same results were also obtained from MBC values of these nanoparticles.

It should be noted that the $\mathrm{pH}$ variations of skin are in the range of 5.5 to 7.4 [39], thus the influence of this parameter on drugs release from MFCSNP and BMCSNNP samples was investigated in these ranges. Figure $4(b-c)$ shows the drugs release profiles of Tre and Clin from BMCSNP and MFCSNP0.05 to AcBS (pH 5.5) and PBS (pH 7.4) during $48 \mathrm{~h}$. As it is evident, a bimodal release behavior was observed regarding the BMCSNP samples along with a rapid rate. An exception trend was seen for the MFCSNP0.05 samples especially those containing Tre which have relative linear drug release profiles. These trends were reasoned due to the high and uniform compactness of the microfluidically generated nanoparticles and resulted in the gradual drugs release along with a gentle slope in the profiles [2]. The other samples showed the profiles with rapid and initial burst release until $30 \mathrm{~h}$. After $30 \mathrm{~h}$, gradual release profiles with gentle slopes were observed for the nanoparticles samples until plateau regimes were occurred for the samples in AcBS [Fig. 4(c)] whereas the drugs release still continued from the samples in PBS environment [Fig. 4(b)]. The first step of the drugs release referred to the erosion of nanoparticles surfaces, which led to the rapid release of drugs molecules from the outer layers of the nanoparticle samples. Whereas, the second step belonged to the slow diffusion of the drugs which entrapped effectively in the inner layers of the nanoparticles based on CS/P(NIPAAm-Co-AA)/CL blend. In general, the larger nanoparticles have a fast release of loaded drugs with respect to those samples with a small size in diameter.

The reason for this behavior was due to the presence of more polymeric layers in the large nanoparticles that the drugs molecules did not enable to diffuse from their surfaces, easily. Nevertheless, different results for the drugs release trends from the MFCSNP0.05 as compared to BMCSNP were observed. The MFCSNP0.05 samples have a sustained controlled release of Tre and Clin drugs compared to BMCSNP samples into PBS. By decreasing the size of 
nanoparticles in MF method, the compactness between the drugs molecules and polymer nanoparticles layers was significantly increased as far as the specific surface area of these nanoparticles was increased, remarkably. These properties of the nanoparticles synthesized by using MF method led to maintaining the drugs molecules through the nanoparticles and their release, slowly.

In conclusion, by considering the drugs release behaviors of the samples in two buffers with different $\mathrm{pH}$, it was observed that the amount and rate of release in AcBS were higher than PBS [Fig. 4(b, c)]. It could be related to the more degradation of the nanoparticles based on CS through AcBS as an acidic medium ( $\mathrm{pH} 5.5$ ) compared to PBS as a natural and physiological environment ( $\mathrm{pH} 7.4)$.

\subsection{Thermal stability study of the samples}

In general, thermal degradations of many drugs encapsulated into polymer carriers are the main drawback. This disadvantage leads to the maintenance of these carriers during long time seems to be difficult and they go through degradation. Therefore, herein, the thermal stability Tre and Clin-loaded BMCSNP and MFCSNP 0.05 was investigated. Based on the residual drugs contents in the samples, this test was done at temperatures of $25 \pm 1^{\circ} \mathrm{C}$ and $45 \pm 1^{\circ} \mathrm{C}$ during thirty days. As it is shown in Fig. 5(a, b), the thermal consistency of drug-loaded MFCSNP0.05 and BMCSNP at temperature of $25^{\circ} \mathrm{C}$ was higher than those samples at $45^{\circ} \mathrm{C}$. At the high temperature, the thermal stability of two drugs specifically Clin molecules has been considered suitable for MFCSNP0.05 samples even after one month because of the complete insertion of the drugs molecules in the nanoparticles bulk in which the nanoparticles layers around the

drugs molecules could protect them from thermal degradation. In contrary, regarding BM technique, the localization of drugs molecules on the nanoparticles samples was superficial that the higher temperature could change the drugs structures followed by their residual contents were significantly decreased.

\subsection{Skin sensitivity, transdermal permeation and residency studies of the samples}


As it was mentioned earlier, the gels containing Tre and Clin drugs were prepared by using a commercialized material namely Carbopol ${ }^{\circledR} 934$ NF. These gel-based materials can adapted themselves to be compatible with different physiological conditions [33].

One of the main disadvantages during the use of Tre as topical formulations is often reddening tracks onto the surface of skin that results in some restrictions to consume it easily for patients. Therefore, we focused on the nanoparticles samples preparation to transfer this drug through the skin layer for elimination of skin irritation without any adverse side effects. Figure 6(a-d) and also Tab. 1(b) demonstrate visually the rabbits' skin inflammation results along with the scoring system for skin sensitivity levels. As compared to the control group (the skin surface received no drugs) [Fig. 6(a)], the use of drugs solution-loaded gel (DS-gel) onto the surface of skin [Fig. 6(b)] showed a severe sensitivity with level score of 3 during $72 \mathrm{~h}$. It means that Tre drug could not transfer thorough the skin layers, as a result, an inflammation was happened in the skin corresponding to a red color. On the other hand, the MFCSNP0.05-gel containing the drugs resulted in a remarkable decrease in inflammation occurrence as far as this adverse observation could be negligible [Fig. 6(d)]. At the end of post-treatment time, by comparing the skin sensitivity levels onto the rabbits' skin, the scores of skin sensitivity happened by the use of BMCSNP-gel and MFCSNP0.05-gel were considered 2 and 1, respectively (see Tab. 1). The reason that the MFCSNP0.05-gel was suitable compared to other samples could be due to the complete firmness between the polymer nanoparticle layers prepared by using MF method and the drugs molecules. Furthermore, the positive charge connection of $\mathrm{NH}^{3+}$ in $\mathrm{CS}$ structure with carboxyl group $\left(\mathrm{COO}^{-}\right)$in Tre structure led a remarkable reduction in the connection between $\mathrm{COO}^{-}$and stratum coreum directly in which the skin sensitivity could not be happened. Consequently, by decreasing in sensitivity as a negative side effect, the skin irritation was significantly declined.

By carrying out this test, it was determined whether the synthesized nanoparticles by using BM and MF techniques enabled to remain through the rats' skins or arrived in the blood circulation, directly. Therefore, the penetration of three samples containing Tre and Clin drugs based on gels in terms of MF-CSNP0.05-gel, BMCSNP-gel and also DS-gel from rat's skins layers was done during $24 \mathrm{~h}$. As it is shown in Figure 7(a), if a suitable interaction was occurred 
between the nanoparticles containing the drugs and hair follicles or stratum coreum, a low permeated drug amount should be observed through the rats' skins. In this way, because of the hydrophilic nature of Clin, it obviously penetrates easily through the inner layers of the skin. Accordingly, this trend could happen in the samples including DS-gel and the permeated drug was decreased by around 15\% using the BMCSNP. By using the MFCSNP0.05-gel, the permeation percentage of Tre and Clin drugs from the skin layers were significantly reduced. Therefore, a large content of these drugs could be remained in the hair follicles and did not arrive at the blood circulation. This amounts of drugs entrapment in the skin layers caused to the drugs delivered effectively to the active sites of acne vulgaris for better efficiency in its treatment. Moreover, it has been tried to by using CS/P(NIPAAm-Co-AAC)/CL blend samples, an increase regarding the drugs storage through follicles reservoirs was happened by increasing in the samples adhesion to hair and however, swelling the nanoparticles near body temperature within the follicles. Due to the uniformity and spherical shape of nanoparticles synthesized by MF method, they were definitely appropriate for topical use as drug delivery system compared to the other samples.

Due to the investigation of expected activity of the drugs based on their permeation through the skin during the predicted time for the treatment period, the skin-stripping study was carried out to investigate the penetration of Tre and Clin in wistar rats' skin layers in different interval times. Figure $7(b, c)$ demonstrates the drugs residency in the skin by using DSgel, BMCSNP and MFCSNP0.05 gels at different time periods. As it can be seen from this figure, the highest drugs amounts were observed regarding the samples including MFCSNP 0.05-gel compared to the others. The reason could be referred to the convenient size and narrow size distribution of nanoparticles and also the adhesion nature of $\mathrm{CS}$ and $\mathrm{CL}$ as well as the temperature and $\mathrm{pH}$ sensitivity of P(NIPAAm-co-AAC) used in nanoparticles structure. Moreover, with more diffusion of the MFCSNP 0.05-gel sample in the hair follicles, the environmental temperature was increased in which the nanoparticles were swollen and as a result, they were entrapped through follicle reservoirs. This phenomenon led to prevent the diffusion of the nanoparticles to the dipper layers of skin as well as blood circulation arrival. The diameters sizes of MFCSNP0.05-gel were adjusted in a range of 200-300 nm in which the 
obtained results showed that this scale was efficient for the sample storage in the hairs follicles [40]. The mutual competition between the positive charge of MFCSNP0.05 and the negative charge of hairs surfaces provided the sample could adhere the hair follicles appropriately while maintaining and releasing the drugs encapsulated by nanoparticles might be occurred as a function of their swelling which led to prevent the adverse side effects of Tre. The study of skin penetration amount and qualitative estimation, the samples were stained by dye in order to use fluorescence microscopy as an efficient tool for investigating the time profile, diffusive streams and diffusive depth of the carriers containing the drugs.

Figure $8(a, b)$ depicts the fluorescence and common lights photographs of the drugs performance in the presence and absence of polymer blend nanoparticles incorporated into the gels containing dye tracer. By comparing two microtomed skin sections, it can be concluded that in contrast to DYDS-gel [Fig. 8(a)], while the DYMFNP0.05-gel was used, the nanoparticles contacting the drugs enabled to permeate extensively through the horny layer and inserted at the desired depth of skin layers which were traced by fluorescence marker [Fig. 8(b)]. Furthermore, the pilosebaceous units owing to the suitable size and adhesion of the nanoparticles have acted as the drugs reservoirs and consequently provided an opportunity that the nanoparticles were able to diffuse into the suitable layers of the skin along with a good stability without arriving in the blood circulation. In other words, these nanocarriers containing the drugs have remained through the skin layers to treat the acne vulgaris [41].

\section{Conclusion}

We successfully prepared two classes of CS-based nanoparticles containing anti-acne drugs by using $\mathrm{BM}$ and MF techniques. The performance evaluations of these samples revealed that the MFCSNP 0.05 sample has the uniform morphology along with the mean diameter size with respect to the sample denoted by BMCSNP. Also, the in vitro release of the drugs showed that the nanoparticles prepared by MF method had the sustained control release of Clin and Tre drugs. The long-term thermal stability of the samples revealed more resistance of MFCSNP samples to the drugs degradation during one month. The animal model experiments on the rabbits and rats' skins demonstrated that using the nanoparticles containing the drugs did not 
have any skin sensitivity or erythema and these nanoparticles due to their engineered average size could enable to penetrate through the skin layers and treat the acne sites. Finally, this research work prospect can apply in the field of transdermal drug delivery by employing these microfluidic-assisted nanoparticles based on CS.

\section{Acknowledgements}

The authors would like to express their sincere thankful from the Iran National Science Foundation (INSF) for full financial support of this project (Contract grant number: 94812762). We would also like to gratitude to research council of University of Tehran for the experimental services.

Declaration of interest: The authors declare that they have no conflict of interest.

\section{References}

[1] R. Karnik, F. Gu, P. Basto, C. Cannizzaro, L. Dean, W. Kyei-Manu, R. Langer, O.C. Farokhzad, Microfluidic platform for controlled synthesis of polymeric nanoparticles, Nano Lett. 8(9) (2008) 2906-2912.

[2] F.S. Majedi, M.M. Hasani-Sadrabadi, J.J. VanDersarl, N. Mokarram, S. Hojjati-Emami, E. Dashtimoghadam, S. Bonakdar, M.A. Shokrgozar, A. Bertsch, P. Renaud, On-chip fabrication of paclitaxel-loaded chitosan nanoparticles for cancer therapeutics, Adv. Funct. Mater. 24(4) (2014) 432-441.

[3] M.M. Hasani-Sadrabadi, S. Taranejoo, E. Dashtimoghadam, G. Bahlakeh, F.S. Majedi, J.J. VanDersarl, M. Janmaleki, F. Sharifi, A. Bertsch, K. Hourigan, L. Tayebi, P. Renaud, K.I. Jacob, Microfluidic manipulation of core/shell nanoparticles for oral delivery of chemotherapeutics: A new treatment approach for colorectal cancer, Adv. Mater. 28 (2016) 4134-4141.

[4] V. Zamora-Mora, D. Velasco, R. Hernández, C. Mijangos, E. Kumacheva, Chitosan/agarose hydrogels: Cooperative properties and microfluidic preparation, Carbohydr. Polym. 111 (2014) 348-355. 
[5] O. Felt, P. Buri, R. Gurny, Chitosan: a unique polysaccharide for drug delivery, Drug Dev. Ind. Pharm. 24(11) (1998) 979-993.

[6] Y. Yang, S. Wang, Y. Wang, X. Wang, Q. Wang, M. Chen, Advances in self-assembled chitosan nanomaterials for drug delivery, Biotechnol. Adv. 32(7) (2014) 1301-1316.

[7] Z. Sun, C. Shi, X. Wang, Q. Fang, J. Huang, Synthesis, characterization, and antimicrobial activities of sulfonated chitosan, Carbohydr. Polym. 155 (2017) 321-328.

[8] T. Şenyiğit, F. Sonvico, S. Barbieri, Ö. Özer, P. Santi, P. Colombo, Lecithin/chitosan nanoparticles of clobetasol-17-propionate capable of accumulation in pig skin, J. Control. Release 142(3) (2010) 368-373.

[9] R. Al-Kassas, J. Wen, A.E.M. Cheng, A.M.J. Kim, S.S.M. Liu, J. Yu, Transdermal delivery of propranolol hydrochloride through chitosan nanoparticles dispersed in mucoadhesive gel, Carbohydr. Polym. 153 (2016) 176-186.

[10] M.A. Ward, T.K. Georgiou, Thermoresponsive polymers for biomedical applications, Polymers 3(3) (2011) 1215-1242.

[11] N. Yu, G. Li, Y. Gao, H. Jiang, Q. Tao, Thermo-sensitive complex micelles from sodium alginate-graft-poly (N-isopropylacrylamide) for drug release, Int. J. Biol. Macromol. 86 (2016) 296-301.

[12] M. Ziaee, P. Zahedi, M. Abdouss, M.A. Zarandi, S. Manouchehri, N. Mozdoori, Electrospun poly ( $\mathrm{N}$-isopropylacrylamide-co-acrylic acid)/cellulose laurate blend nanofibers containing adapalene: Morphology, drug release, and cell culture studies, Int. J. Polym. Mater. 65(9) (2016) 477-486.

[13] M. Hamidi, A. Azadi, P. Rafiei, Hydrogel nanoparticles in drug delivery, Adv. Drug Deliver. Rev. 60(15) (2008) 1638-1649.

[14] S. Chen, H. Zhang, X. Shi, H. Wu, N. Hanagata, Microfluidic generation of chitosan/CpG oligodeoxynucleotide nanoparticles with enhanced cellular uptake and immunostimulatory properties, Lab Chip 14(11) (2014) 1842-1849.

[15] S.L. Hedden, S. Davidson, C.B. Smith, Cause and effect: The relationship between acne and self-esteem in the adolescent years, JNP-J. Nurse Pract. 4(8) (2008) 595-600. 
[16] J. Koo, The psychosocial impact of acne: patients' perceptions, J. Am. Acad. Dermatol. 32(5) (1995) S26-S30.

[17] R.J. Lasek, M.M. Chren, Acne vulgaris and the quality of life of adult dermatology patients, Arch. Dermatol. 134(4) (1998) 454-458.

[18] R. Berger, R. Rizer, A. Barba, D. Wilson, D. Stewart, R. Grossman, M. Nighland, J. Weiss, Tretinoin gel microspheres $0.04 \%$ versus $0.1 \%$ in adolescents and adults with mild to moderate acne vulgaris: a 12-week, multicenter, randomized, double-blind, parallel-group, phase IV trial, Clin. Ther. 29(6) (2007) 1086-1097.

[19] S.F. Friedlander, L.F. Eichenfield, J.F. Fowler Jr, R.G. Fried, M.L. Levy, G.F. Webster, Acne epidemiology and pathophysiology, Seminars in cutaneous medicine and surgery, WB Saunders, 2010, pp. 2-4.

[20] A. Krautheim, H.P.M. Gollnick, Acne: topical treatment, Clin. Dermatol. 22(5) (2004) 398407.

[21] D. Thiboutot, H. Gollnick, V. Bettoli, B. Dréno, S. Kang, J.J. Leyden, A.R. Shalita, V.T. Lozada, D. Berson, A. Finlay, New insights into the management of acne: an update from the global alliance to improve outcomes in acne group, J. Am. Acad. Dermatol. 60(5) (2009) S1-S50.

[22] J.J. Leyden, A review of the use of combination therapies for the treatment of acne vulgaris, J. Am. Acad. Dermatol. 49(3) (2003) S200-S210.

[23] J.S. Weiss, Current options for the topical treatment of acne vulgaris, Pediatr. Dermatol. 14(6) (1997) 480-488.

[24] O.H. Mills Jr, A.M. Kligman, Treatment of acne vulgaris with topically applied erythromycin and tretinoin, Acta Derm.-Venereol. 58(6) (1977) 555-557.

[25] R.L. Rietschel, S.H. Duncan, Clindamycin phosphate used in combination with tretinoin in the treatment of acne, Int. J. Dermatol. 22(1) (1983) 41-43.

[26] T.J. Franz, P.A. Lehman, Pharmacology of retinoids in the skin, in: U. Reichert, B. Shroot (Eds.), Pharmacology and the skin, KARGER Medical and Scientific Publishers, Cannes, France, 1989, pp. 174-180. 
[27] R. Anadolu, T. Sen, N. Tarimci, A. Birol, C. Erdem, Improved efficacy and tolerability of retinoic acid in acne vulgaris: a new topical formulation with cyclodextrin complex $\psi$, J. Eur. Acad. Dermatol. 18(4) (2004) 416-421.

[28] M. Manconi, D. Valenti, C. Sinico, F. Lai, G. Loy, A.M. Fadda, Niosomes as carriers for tretinoin: II. Influence of vesicular incorporation on tretinoin photostability, Int. J. Pharm. 260(2) (2003) 261-272.

[29] K.A. Shah, A.A. Date, M.D. Joshi, V.B. Patravale, Solid lipid nanoparticles (SLN) of tretinoin: potential in topical delivery, Int. J. Pharm. 345(1) (2007) 163-171.

[30] C. Sinico, M. Manconi, M. Peppi, F. Lai, D. Valenti, A.M. Fadda, Liposomes as carriers for dermal delivery of tretinoin: in vitro evaluation of drug permeation and vesicle-skin interaction, J. Control. Release 103(1) (2005) 123-136.

[31] L. Romoli, G. Tantussi, G. Dini, Experimental approach to the laser machining of PMMA substrates for the fabrication of microfluidic devices, Opt. Laser. Eng. 49(3) (2011) 419-427.

[32] M. Tarameshlou, S.H. Jafari, I. Rezaeian, H.A. Khonakdar, A microfluidic approach to synthesize monodisperse poly (2-hydroxyethyl methacrylate) based spherical microgels via water in water emulsion technique, Int. J. Polym. Mater. 63(17) (2014) 884-890.

[33] S.C. Shin, H.J. Kim, I.J. Oh, C.W. Cho, K.H. Yang, Development of tretinoin gels for enhanced transdermal delivery, Eur. J. Pharm. Biopharm. 60(1) (2005) 67-71.

[34] E. Nester, D. Anderson, J.C.E. Roberts, M. Nester, Microbiology: A human perspective, 6 ed., McGraw-Hill New York, 2008.

[35] M.R. Avadi, A.M.M. Sadeghi, A. Tahzibi, K.H. Bayati, M. Pouladzadeh, M.J. Zohuriaan-Mehr, M. Rafiee-Tehrani, Diethylmethyl chitosan as an antimicrobial agent: Synthesis, characterization and antibacterial effects, Eur. Polym. J. 40(7) (2004) 1355-1361.

[36] W.L. Du, S.S. Niu, Y.L. Xu, Z.R. Xu, C.L. Fan, Antibacterial activity of chitosan tripolyphosphate nanoparticles loaded with various metal ions, Carbohydr. Polym. 75(3) (2009) 385-389.

[37] L. Qi, Z. Xu, X. Jiang, C. Hu, X. Zou, Preparation and antibacterial activity of chitosan nanoparticles, Carbohyd. Res. 339(16) (2004) 2693-2700. 
[38] J.H. Draize, G. Woodard, H.O. Calvery, Methods for the study of irritation and toxicity of substances applied topically to the skin and mucous membranes, J. Pharmacol. Exp. Ther. 82(3) (1944) 377-390.

[39] M.H. Schmid-Wendtner, H.C. Korting, The $\mathrm{pH}$ of the skin surface and its impact on the barrier function, Skin Pharmacol. Phys. 19(6) (2006) 296-302.

[40] H.N. Huang, T.L. Li, Y.L. Chan, C.L. Chen, C.J. Wu, Transdermal immunization with lowpressure-gene-gun mediated chitosan-based DNA vaccines against Japanese encephalitis virus, Biomaterials 30(30) (2009) 6017-6025.

[41] P.M. Elias, Epidermal lipids, barrier function, and desquamation, J. Invest. Dermatol. 80 (1983) 44-49.

\section{Figures caption}

Fig. 1. Schematic representation of MF device used: (a) the channel and bottom layers made from PMMA along with the channels dimensions, (b) the inlets and outlet for fluids flows and (c) hydrodynamic flow focusing regions at three different rhodamine $\mathrm{B} /$ sodium fluorescein ratios of $0.05,0.1$ and 0.2 .

Fig. 2. The DLS results including diameter and size distribution and the SEM micrograph images of CS/P(NIPAAm-Co-AAc)/CL (6:1:1) nanoparticles: (a) BMCSNP obtained at non-microfluidic condition and (b) to (d) which resulted at different solutions fluid flows ratios of $0.05,0.1$ and 
0.2, respectively, (e) bulk mixing (BM) method and (f) microfluidic (MF) technique corresponding to the sample denoted by MFCSNPs 0.05 (the scale bar is 1 micron).

Fig. 3. (a) The loading efficiency of Clin and Tre drugs and (b) the positive charge on the BMCSNP and MFCSNP with different flow rates ratios. Up: one and two stars stand for $p$ value $>0.05$ and p-value $<0.01$, respectively. Down: the data with $p$-values $<0.01$.

Fig. 4. The antibacterial evaluation of the samples against P.acnes by using disc diffusion method after $24 \mathrm{~h}$ : (1) DS, (2) BMCSNP without drugs, (3) MFCSNP0.05 without drugs, (4) BMCSNP with drugs, and (5) MFCSNP0.05 with drugs (a), and the drugs release profiles of BMCSNP and MFCSNP0.05 during $48 \mathrm{~h}$, at $\mathrm{pH} 7.4$ (b) and $\mathrm{pH} 5.5$ (c).

Fig. 5. The thermal stability of Tre and Clin drugs molecules loaded into BMCSNP and MFCSNP0.05 samples at different temperatures (a) $25^{\circ} \mathrm{C}$ and (b) $45^{\circ} \mathrm{C}$ during thirty days.

Fig. 6. The skin sensitivity photographs of rabbit's skin by using different samples containing Tre and Clin drugs after $72 \mathrm{~h}$ post-treatment time: (a) control, (b to d) treated with DS-gel, BMCSNP-gel and MFCSNPO.05-gel, respectively.

Fig. 7. (a) The permeated Tre and Clin drugs amounts into the rats' skin layers by using the DSgel, BMCSNP and MFCSNP0.05 gels, (b) the amounts of Clin and (c) Tre drugs remained in the rats' skin layers by using the samples including DS-gel, BMCSNP and MFCSNP0.05-gels during 24 h post-treatment time.

Fig. 8. The florescence and common lights photographs of the rat's microtomed skin treated with (a) DYDS-gel and (b) DYMFCSNP0.05-gel. (The blue arrows are the dye tracer streams which showed the MFCSNP 0.05 could penetrate successfully through the skin layers). 
(a)

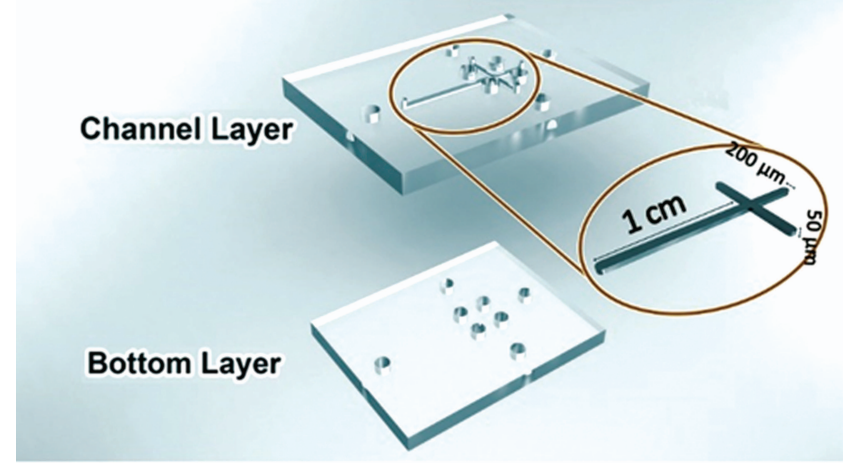

(b)

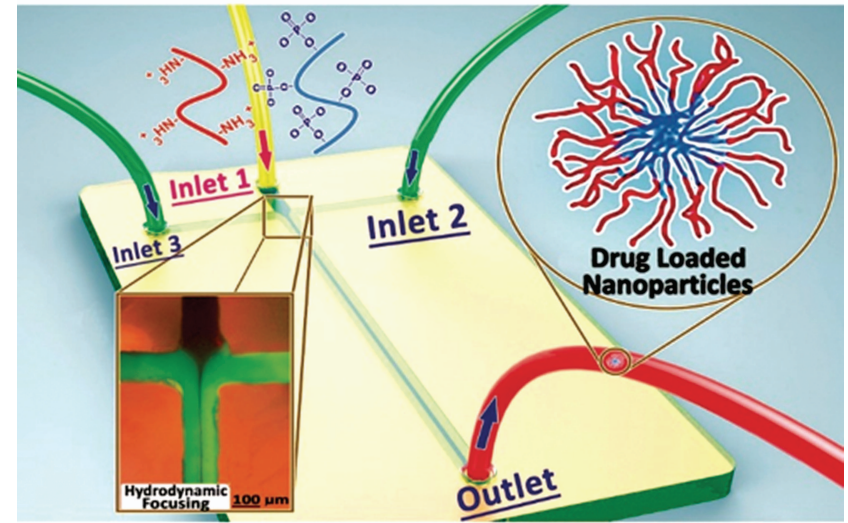

(c)

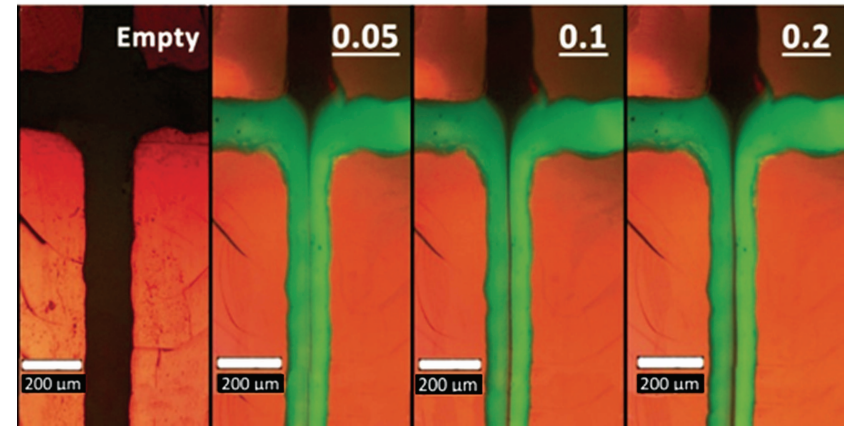



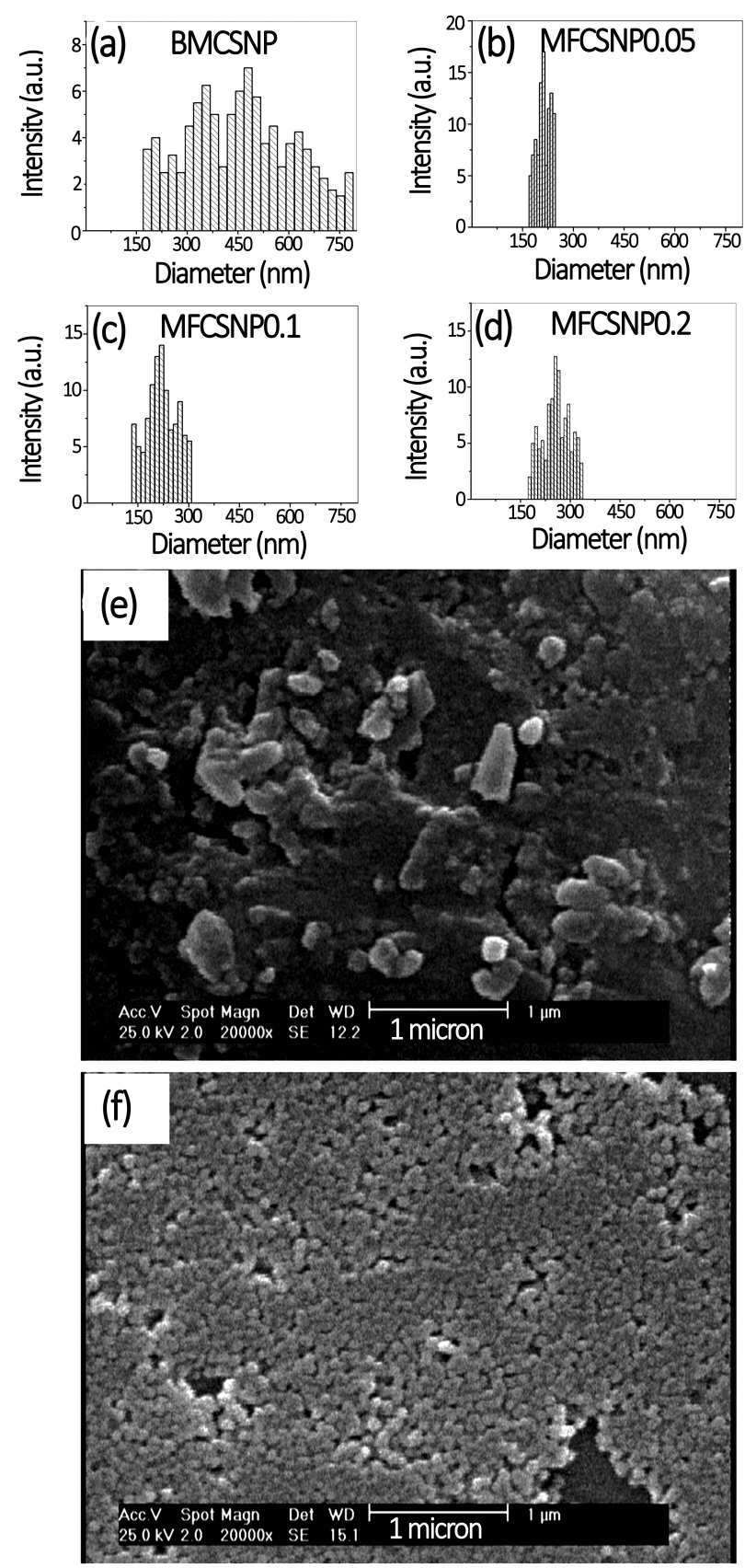

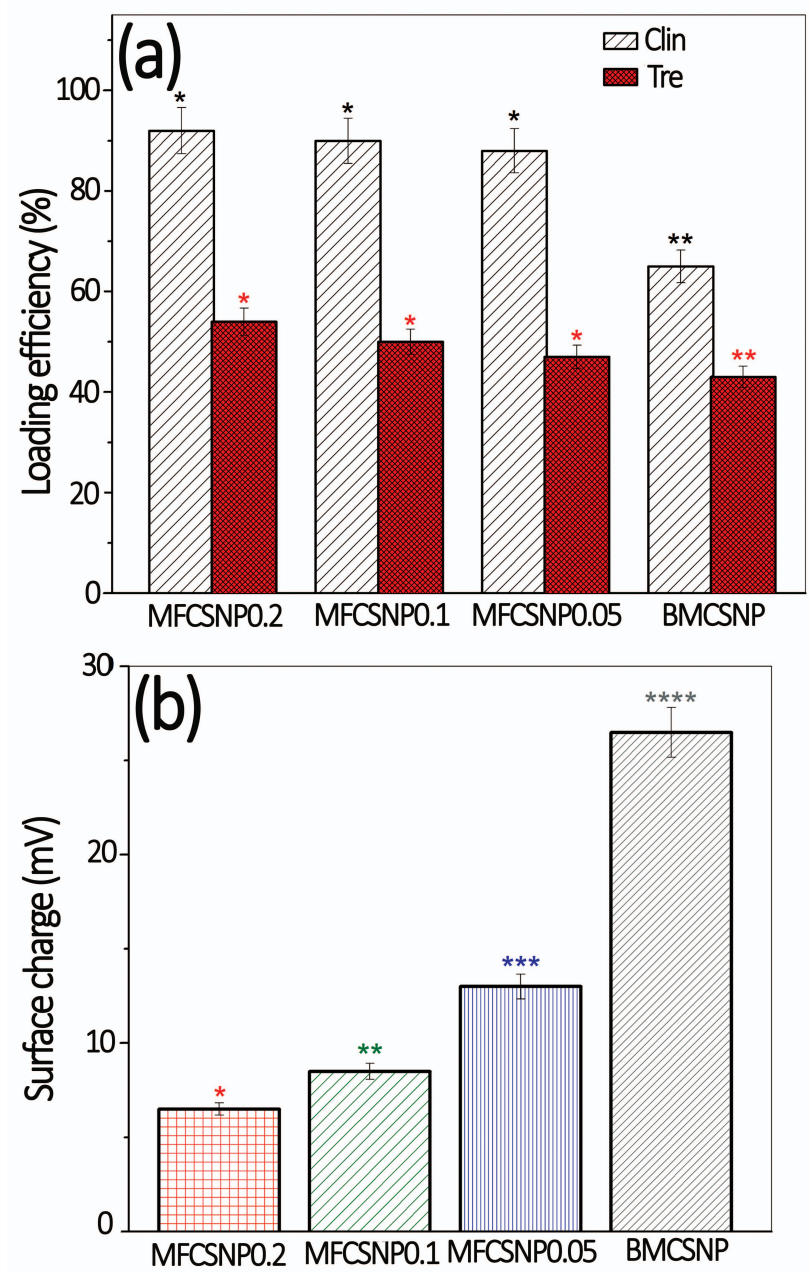

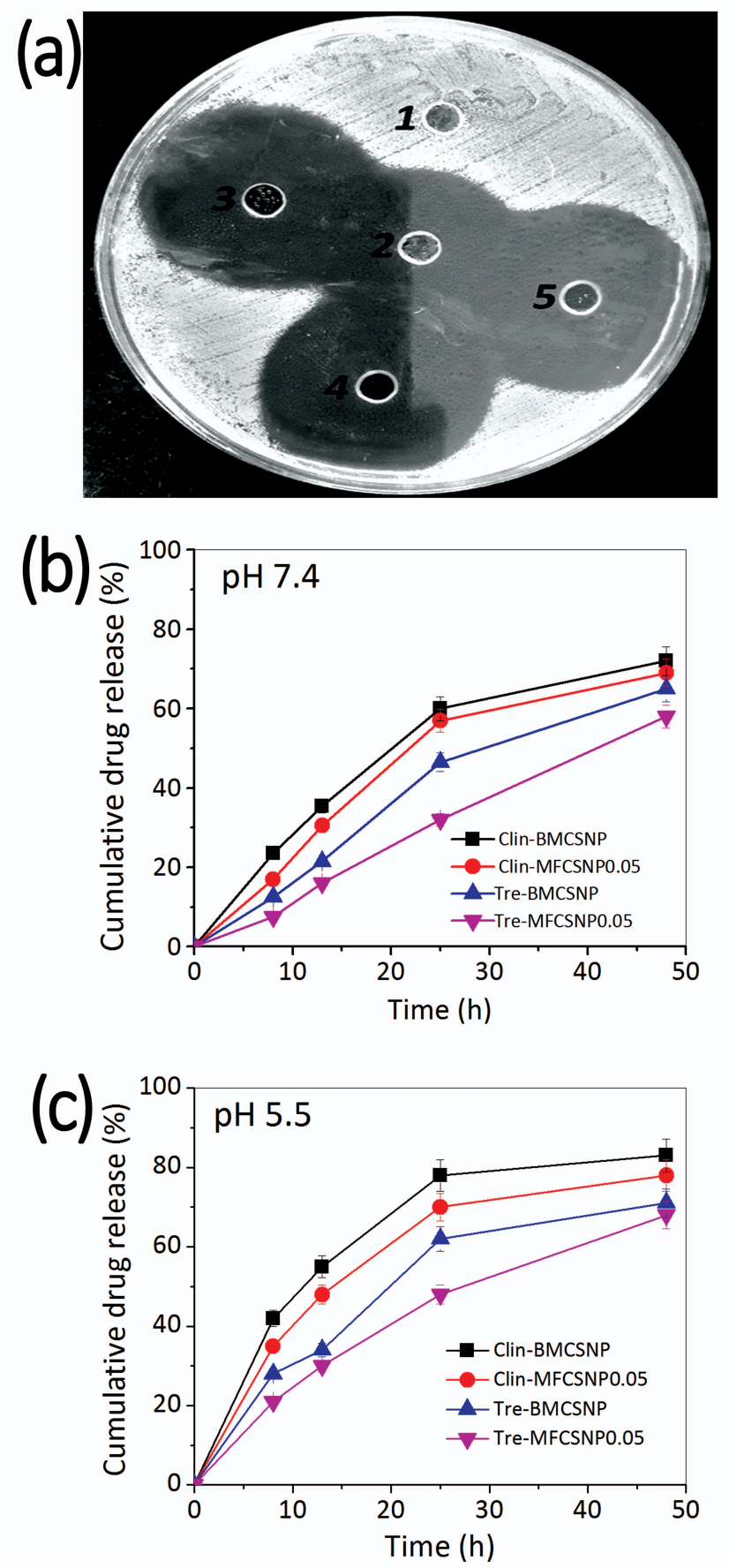

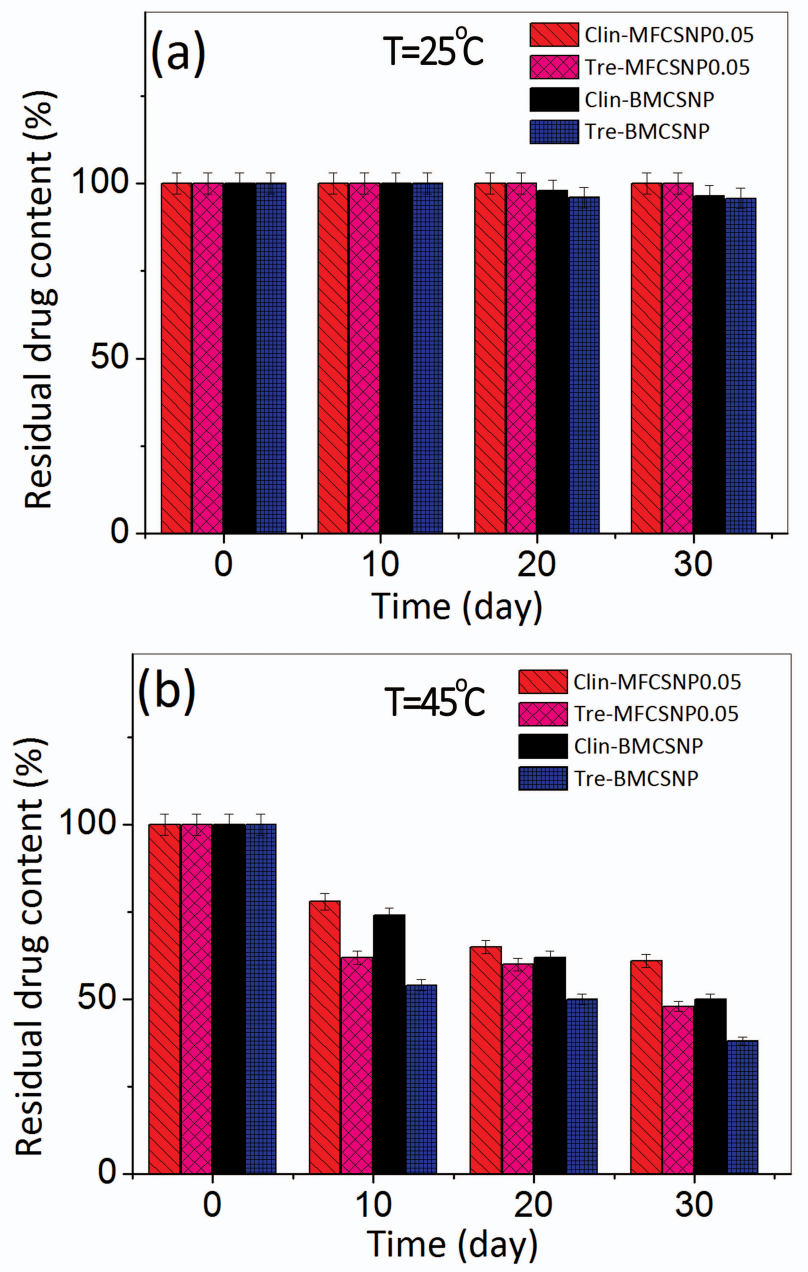

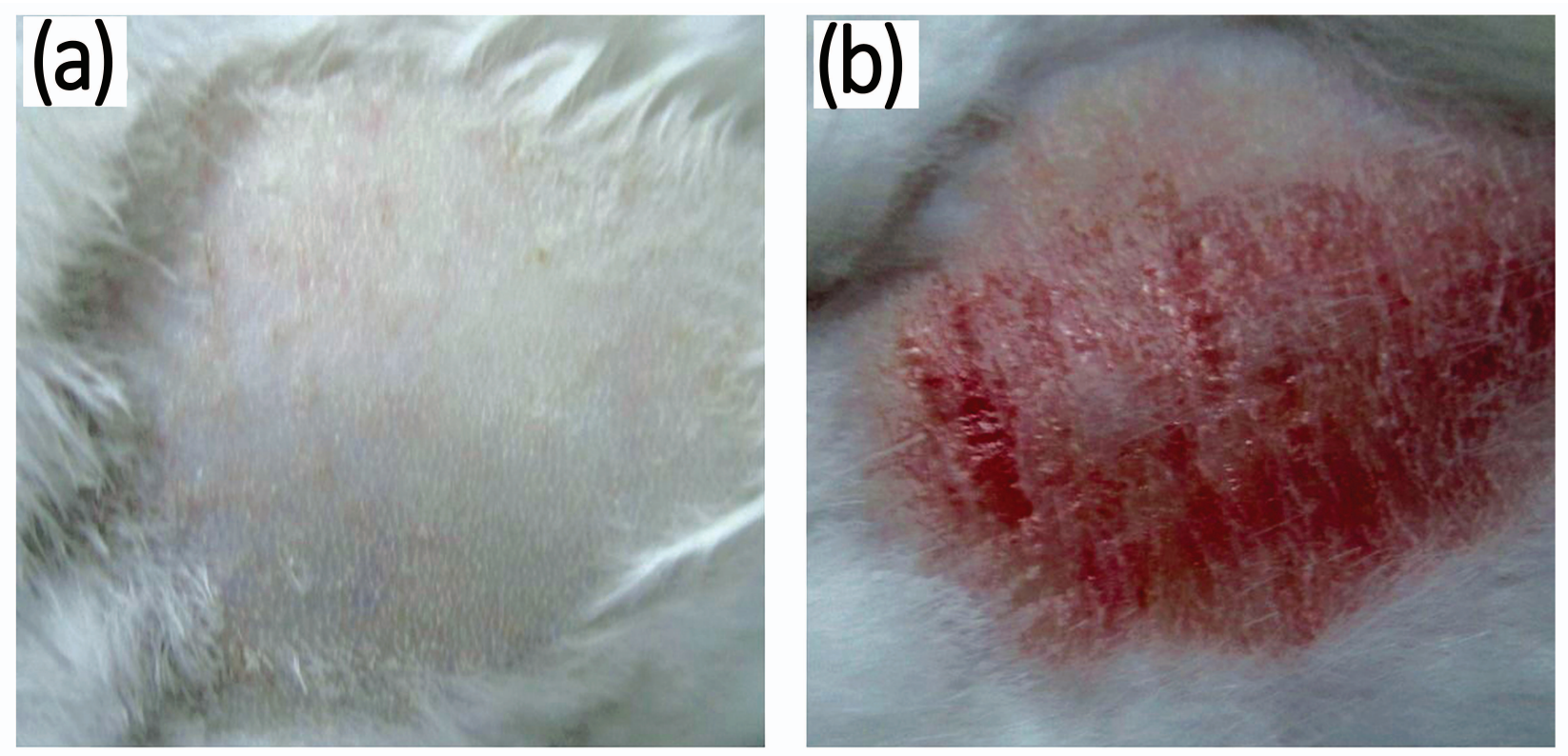

(c)

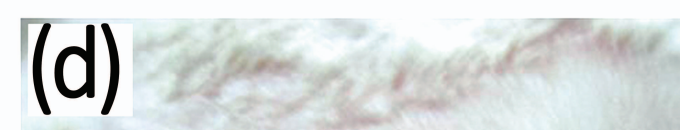
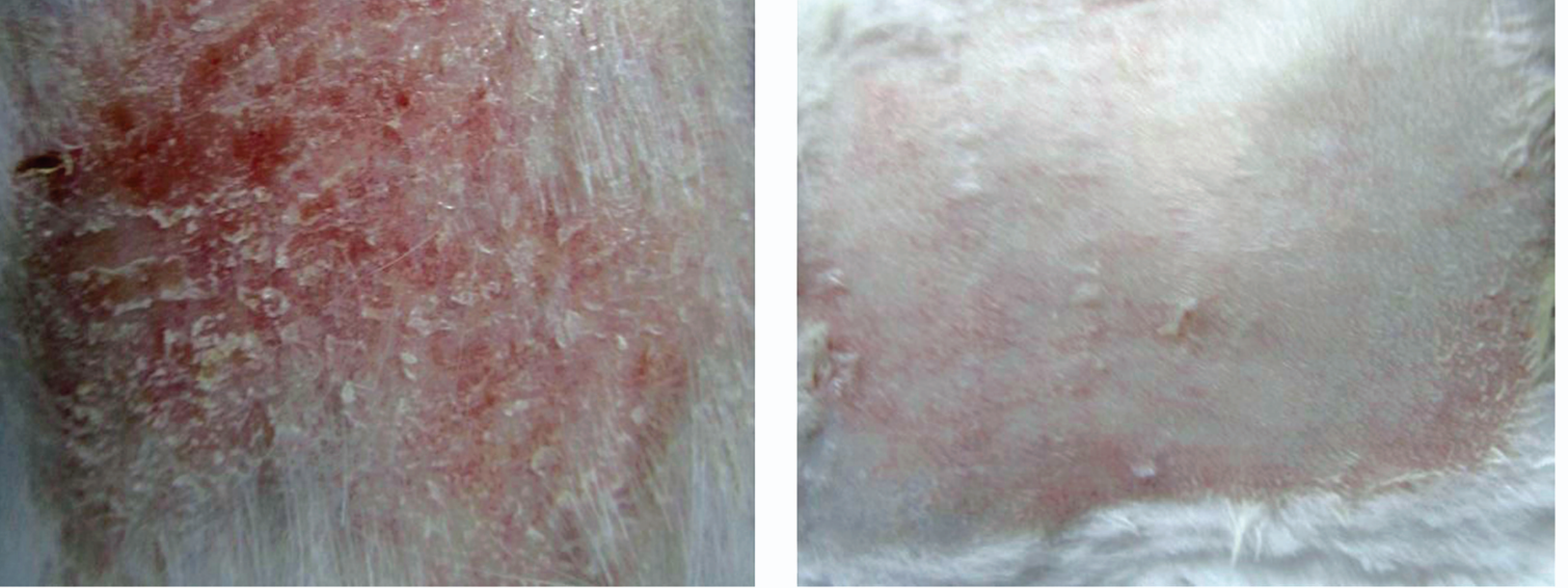

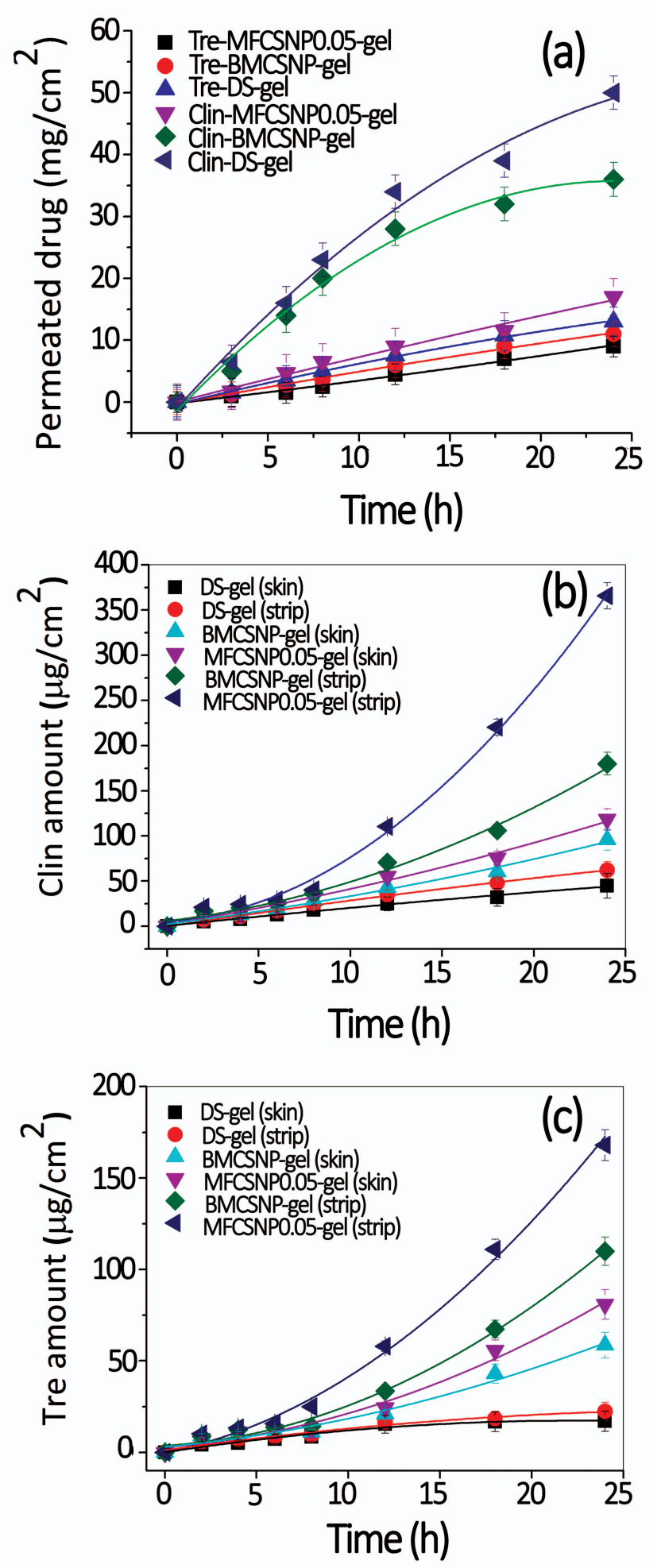


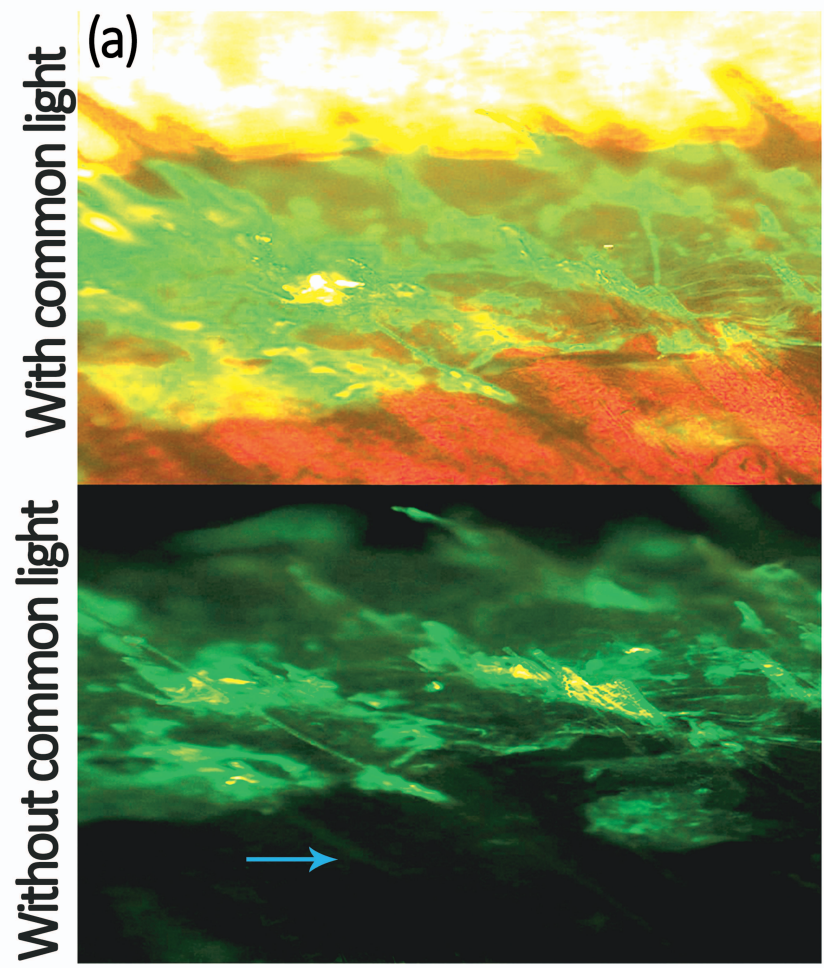

(b)

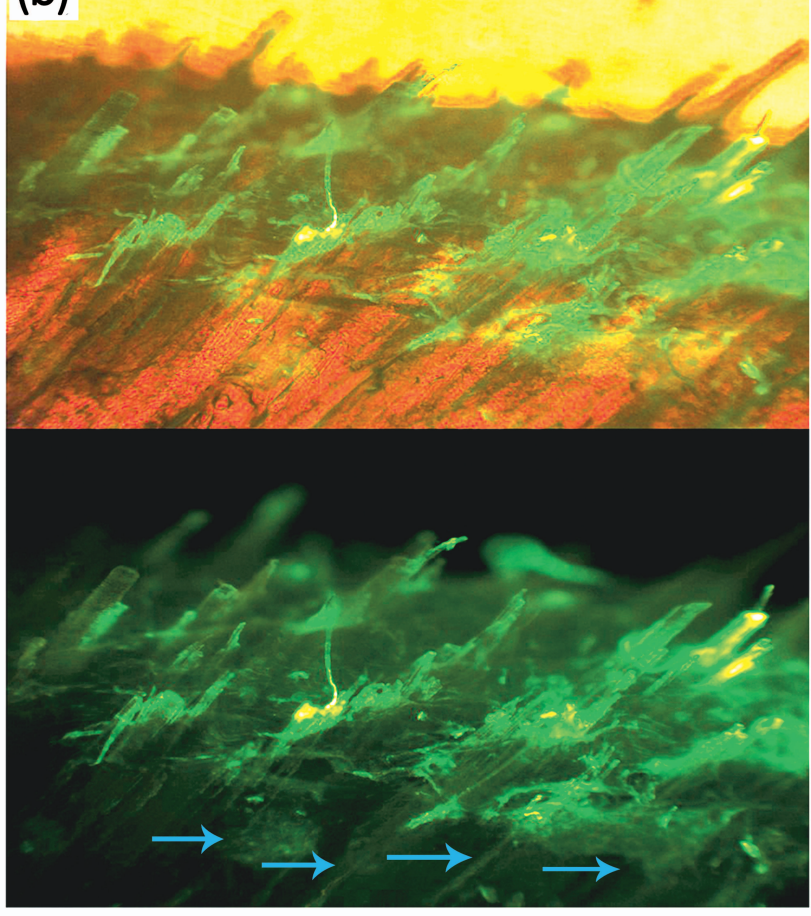


Table 1. (a) The MIC and MBC values of the different samples including DS, BMCSNP and MFCSNP0.05 in the presence and absence of Tre and Clin drugs by using the antibacterial test, and (b) the sensitivity levels of rabbit's skins in four groups treated with no drugs (control), DSgel, BMCSNP-gel and MFCSNP0.05-gel based on the scoring system including 0, 1, 2, and 3 corresponding to no color, light pink-color, dark pink-color and red-color, respectively during 72 h post-treatment time.

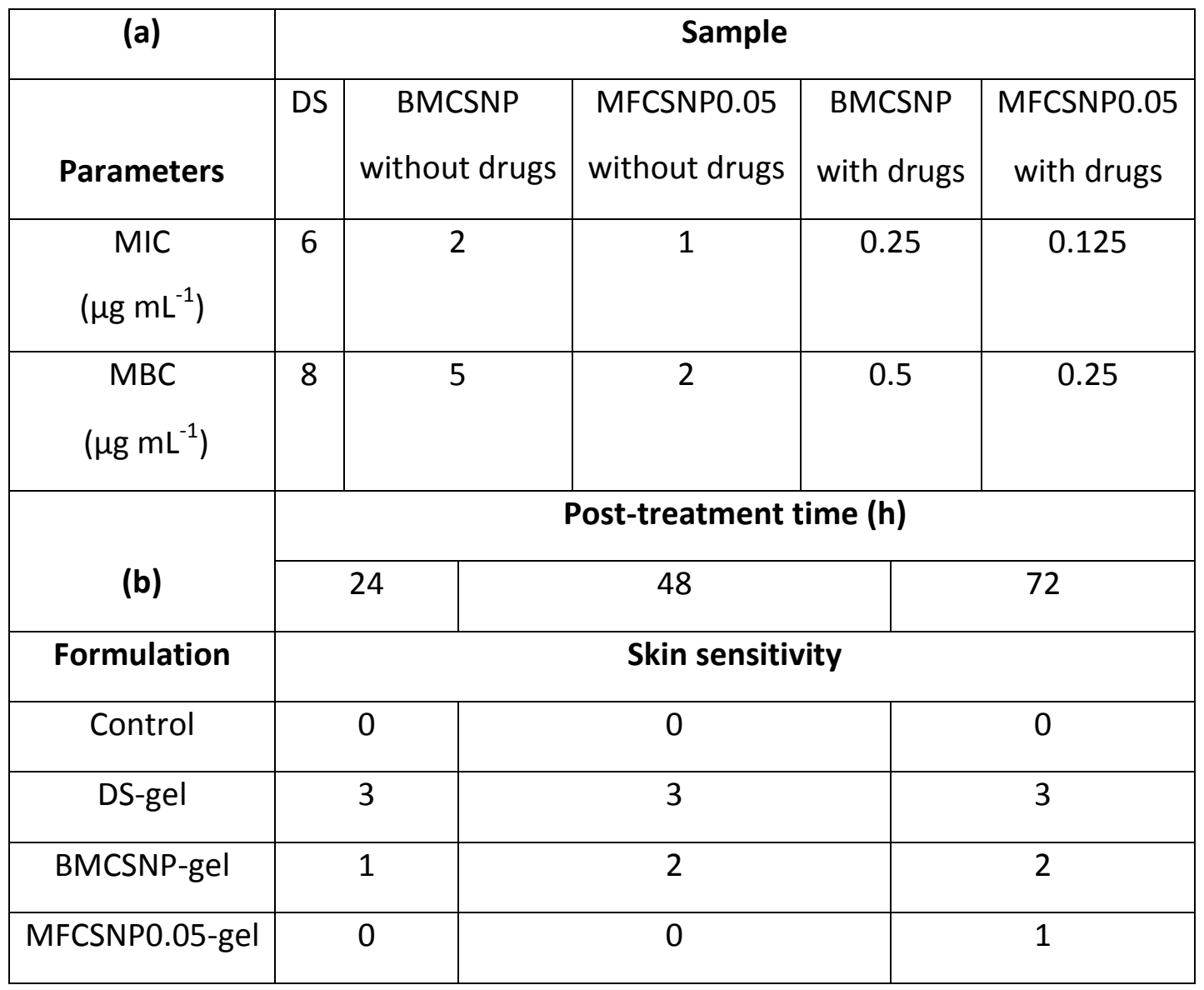

